\title{
The molecular genetics of the human $I$ locus and molecular background explain the partial association of the adult i phenotype with congenital cataracts
}

\author{
Lung-Chih Yu, Yuh-Ching Twu, Ming-Lun Chou, Marion E. Reid, Alan R. Gray, Joann M. Moulds, Ching-Yi Chang, and Marie Lin
}

\begin{abstract}
The human $i$ and $I$ antigens are characterized as linear and branched repeats of $\mathrm{N}$-acetyllactosamine, respectively. Conversion of the $i$ to the I structure requires I-branching $\beta-1,6-N$-acetylglucosaminyltransferase activity. It has been noted that the null phenotype of $I$, the adult i phenotype, is associated with congenital cataracts in Asians. Previously, the identification of molecular changes in the IGnT gene, associated with the adult i phenotype, has been reported. In the present study, we demonstrate that the human I locus expresses 3 IGnT forms, desig-
\end{abstract}

nated IGnTA, IGnTB, and IGnTC, which have different exon 1, but identical exons 2 and 3 , coding regions. The molecular genetics proposed for the Illocus offer a new perspective on the formation and expression of the I antigen in different cells and provide insight into the questions derived from investigation of the adult i phenotype. Molecular genetic analyses of the I loci of the 2 adult i groups, with and without congenital cataracts, were performed, and enzyme function assays and expression patterns for the 3 IGnT transcripts in reticulocytes and lens- epithelium cells were analyzed. The results suggest a molecular genetic mechanism that may explain the partial association of the adult i phenotype with congenital cataracts and indicate that a defect in the I locus may lead directly to the development of congenital cataracts. The results also suggest that the human blood group I gene should be reassigned to the IGnTC form, not the IGnTB form, as described previously. (Blood. 2003;101: 2081-2088)

(C) 2003 by The American Society of Hematology

\section{Introduction}

The $\mathrm{i}$ and I antigens are carbohydrate structures characterized as linear and branched repeats of $N$-acetyllactosamine, Gal $\beta 1$ 4GlcNAc $\beta 1-3$ Gal $\beta 1-4 G l c N A c-R$ and Gal $\beta 1-4 G l c N A c \beta 1-3(G a l \beta 1-$ 4 GlcNAc $\beta 1-6)$ Gal $\beta 1-4 G l c N A c-R$, respectively, carried on glycolipids and glycoproteins. ${ }^{1-5}$ They were first identified on human red blood cells (RBCs), ${ }^{6-10}$ but are known to be present also on the surface of most human cells and on soluble glycoproteins in various body fluids, including milk, ${ }^{11}$ saliva, ${ }^{12}$ plasma, ${ }^{12}$ amniotic fluid, urine, and ovarian cyst fluid. ${ }^{4,13}$ The $N$-acetyllactosamine repeats are synthesized by the sequential action of $\beta-1,3-N$ acetylglucosaminyltransferase and $\beta$-1,4-galactosyltransferase. Conversion of the $i$ antigen into an I-active structure requires the action of a third enzyme, the I-branching $\beta-1,6-N$-acetylglucosaminyltransferase (I $\beta 6 \mathrm{GlcNAcT}){ }^{2,14,15}$ It was determined that the expression of the I and i antigens reflects a reciprocal relationship that is developmentally regulated. Adult human RBCs fully express I antigens and contain only a few $\mathrm{i}$ antigens, which predominate in fetal and neonatal RBCs. After birth, the quantity of I antigen gradually increases as the level of $i$ antigen falls, until the healthy adult RBC Ii status is reached at about 18 months of life. ${ }^{10,16}$ The developmentally regulated expression of Ii antigen is exhibited on

From the Transfusion Medicine Laboratory and the Immunohematology Reference Laboratory, Mackay Memorial Hospital, and the Institute of Biochemical Sciences, College of Science, National Taiwan University, Taipei, Taiwan; the Immunohematology Laboratory, New York Blood Center, New York, NY; the National Blood Service-Tooting, United Kingdom; and the Department of Microbiology and Immunology, Drexel University College of Medicine, Philadelphia, PA.

Submitted September 10, 2002; accepted October 18, 2002. Prepublished online as Blood First Edition Paper, November 7, 2002; DOI 10.1182/blood2002-09-2693.

Supported in part by National Health Research Institute grant NHRI-EX908601SL (M.L.) and National Science Council grant NSC 91-2314-B-002-405
RBCs and also in many other tissues. Furthermore, altered expression patterns for the I and $\mathrm{i}$ antigens have often been observed during oncogenesis ${ }^{17}$ and, thus, the Ii are considered oncodevelopmental antigens. ${ }^{18}$

Most adult RBCs fully express the I antigen; however, in a small percentage of individuals, the RBCs are rich in the $\mathrm{i}$ antigen and contain very low levels of I.9,10,16 This phenotype is called the adult i phenotype and is believed to result from lack of I-branching transferase activity. ${ }^{3,14}$ Although the adult i phenotype is rare, it has attracted considerable attention because it has been noted to be associated with congenital cataracts in Asians. Yamaguchi et al ${ }^{19}$ first reported the association of the adult $i$ phenotype with congenital cataracts among Japanese individuals. ${ }^{20,21}$ Of 31 Japanese with the adult i phenotype, 29 had congenital cataracts, whereas none of the I-phenotype members of these families had the same eye defect, indicating that there was no recombination between the Ii phenotype and cataracts. Linkage of the adult $\mathrm{i}$ phenotype and congenital cataracts has been observed in 3 Taiwanese pedigrees. ${ }^{22,23}$ In these 3 families, the 5 i members all have congenital cataracts, whereas none of the other 17 members with the I phenotype are so afflicted. The association does not seem

and National Research Program for Genomic Medicine of National Science Council grant NSC 91-3112-B-002-028 (L.-C.Y.).

The online version of the article contains a data supplement.

Reprints: Marie Lin, Transfusion Medicine Laboratory, Mackay Memorial Hospital, 45 Ming-San Rd, Tamshui, Taipei County 251, Taiwan; e-mail: marilin@ms2.mmh.org.tw.

The publication costs of this article were defrayed in part by page charge payment. Therefore, and solely to indicate this fact, this article is hereby marked "advertisement" in accordance with 18 U.S.C. section 1734.

(C) 2003 by The American Society of Hematology 
to be as pronounced in the white population, however, with only 3 individuals in 2 families formally reported as having both the adult i phenotype and congenital cataracts. ${ }^{24-26}$

The association of the adult $\mathrm{i}$ phenotype with congenital cataracts can be explained either by a close linkage between independent I- and cataract-related genes or by a pleiotropic effect of the gene responsible for the adult i phenotype on the development of cataracts. However, because of the reduced strength of the association in whites, it has been suggested that the former hypothesis of a close linkage between 2 independent genes is the more tenable one. ${ }^{24}$

We have previously reported molecular genetic analysis of the adult i phenotype in Taiwanese subjects who also suffered from congenital cataracts. ${ }^{23}$ The results obtained from mutation detection within the 2 I-branching enzyme-encoding genes, segregation analyses, and enzyme function assays have identified an association between molecular changes in the IGnT gene and the adult $\mathrm{i}$ phenotype. These findings suggest that the $I G n T$ gene, first reported in 1993 by Bierhuizen et al, ${ }^{27}$ is the candidate for the blood group $I$ gene. Further, it was demonstrated that 3 different molecular bases, including 2 missense mutations (1043G $>$ A and $1148 \mathrm{G}>\mathrm{A})$ and a gene deletion, are responsible for the adult i phenotype. ${ }^{23}$ Despite the identification of the molecular bases for the adult i phenotype, the molecular genetic basis for the association of the adult $i$ phenotype with congenital cataracts remains obscure. The molecular factors revealed indicate, however, that the association may result from a pleiotropic effect of the same gene mutation but not from a linkage of 2 independent genes, because it is unlikely that 2 different nucleotide changes would be linked to the nearby gene that has, by chance, also mutated to result in the development of cataracts.

The present investigation shows not only that the human $I$ locus expresses the previously reported $I G n T$ transcript, but also that 3 $I G n T$ forms are expressed from the human $I$ locus. Comparison of molecular genetic analyses for adult i Taiwanese and white subjects, with and without congenital cataracts, respectively, reveals a molecular genetic background accounting for the partial association of the adult i phenotype with congenital cataracts and redefines the $I G n T$ gene form responsible for the expression of the blood group I antigen on RBCs.

\section{Patients, materials, and methods}

\section{Samples}

Our use of human subjects was conducted under the tenets of the Helsinki protocol, and the program was approved by the Institutional Review Board at Mackay Memorial Hospital. Peripheral blood or genomic DNA samples were obtained from 6 unrelated whites bearing the adult i phenotype, denoted as JCC, 97013, 987U1, 944C2, 1110Y2, and 1110X3. The 5 samples from Taiwanese with the adult i phenotype were designated as W-3, W-5, S-6, S-7, and C-3, and have been described previously. ${ }^{23}$ The 5 adult 1 Taiwanese all have congenital cataracts, whereas the 6 adult $i$ whites are not so afflicted. The QIAamp DNA Blood Mini Kit (Qiagen GmbH, Hilden, Germany) and the QIAamp RNA Blood Mini Kit (Qiagen) were used to purify genomic DNA and total RNA, respectively, from the peripheral blood cells. Genomic DNA from 51 randomly selected whites was prepared from their saliva using the QIAamp DNA Blood Mini Kit.

Poly $\mathrm{A}^{+}$RNA samples from the human brain (cerebellum), heart, small intestine, kidney, and prostate were purchased from Clontech Laboratories (Palo Alto, CA).

\section{Demonstration of the existence and structures of 3 IGnT transcripts: IGnTA, IGnTB, and IGnTC}

The $5^{\prime}$ and $3^{\prime}$ rapid amplification of cDNA end (RACE) was performed on Marathon-Ready cDNA derived from human prostate (Clontech) to establish the cDNA structures of the IGnTA, IGnTB, and IGnTC transcripts. Primers used were as follows: IR32 (ATGTACATAGTGGCCGTGGCAGCCTCC, antisense sequence, spanning the exons 2-3 junction of the 3 IGnT cDNAs), IAR34 (5'-ACGCGCTAAAAAGACAGTGCTTCCAAGAGC$3^{\prime}$, for IGnTA, complementary of nucleotides 8-37, codon for initiation methionine as nucleotides 1-3), IBR35 (5'-TGTGCAAACTTGAGTCAGCCTCAAAGGGTC-3', for IGnTB, complementary of nucleotides 118-147), ICR36 (5'-CTCACAAAAAGACCACGCTGAGCAGAGTG-3', for IGnTC, complementary of nucleotides 33-62) for 5'-RACE, IF23 (5'-GGAGGCTGCCACGGCCACTATGTACATGG-3', spanning the exons 2-3 junction), and IFa (5'-CAGAGTGAAACTGCGATACAACCCAGCTGG-3', 7 nucleotides upstream to the stop codon in exon 3) for $3^{\prime}$-RACE. Amplified DNA fragments from RACE were cloned into the pCRII-TOPO vectors by a TOPO TA Cloning Kit (Invitrogen, Groningen, The Netherlands). The DNA sequences were determined using the BigDye Terminator Cycle Sequencing Kit (Applied Biosystems, Foster City, CA). The full cDNA structures of the 3 IGnT forms were built by assembling the sequences from the longest $5^{\prime}$ - and $3^{\prime}$-RACE products, and the full cDNA structures were further demonstrated by reverse transcription polymerase chain reaction (RT-PCR) using the synthetic primers that anneal to the most $5^{\prime}$ and $3^{\prime}$ sequences of the cDNAs.

\section{RT-PCR analysis for the expression of IGnTA, IGnTB, and IGnTC}

RT-PCR was used to evaluate the expression of the $3 \mathrm{IGnT}$ transcripts for different human tissues and cells. The first-strand cDNAs were primed by oligo-dT primer and synthesized by ThermoScript reverse transcriptase (Invitrogen). PCR amplification was then performed, using the forward primers IAF1 (5'-GGAGCCACTTCAGAAATGTGTCAC-3', for IGnTA, nucleotides -236 through -213 , codon for initiation methionine as nucleotides 1-3), IBFm (5'-GATGAAACGGAATCGATTCCCAGCGTCTCC-3', for IGnTB, nucleotides -147 through -118), and ICF2 (5'-GCAAATTCAACCTCTCACACCGATC-3', for IGnTC, nucleotides -76 through -52$)$. The reverse primer, IRc ( $5^{\prime}$-AGCTGCAGTTTCCCTTCAGTCATGAGTAGC-3', antisense sequence, 12 nucleotides downstream to the stop codon in exon 3 ), was common for the 3 transcripts. The PCR program included 5 minutes at $94^{\circ} \mathrm{C}$ followed by 40 cycles of 30 seconds at $94^{\circ} \mathrm{C}, 30$ seconds at $62^{\circ} \mathrm{C}$, and 1.5 minutes at $72^{\circ} \mathrm{C}$.

\section{Molecular cloning and sequence analysis for the IGnT gene of the adult $\mathrm{i}$ individuals}

The exon 1A, 1B, 1C, 2, and 3 regions of the $I G n T$ gene were amplified by PCR using genomic DNA as a template. The primer pairs were as follows: IAF1 and IAR5 (5'-TAGTATTGACCATGCAGTGTTTATTCG-3', antisense sequence, 30 nucleotides downstream to exon 1A) for exon $1 \mathrm{~A}$, IBFm and IBRd (5'-CCTAATAAAAAGTGGCTGGTTATTCTAAAGCC-3', antisense sequence, 50 nucleotides downstream to exon $1 \mathrm{~B}$ ) for exon $1 \mathrm{~B}$, ICF2 and ICR6 (5'-TGAGTCAGTTCTCTAGGCGAGCAG-3', antisense sequence, 49 nucleotides downstream to exon 1C) for exon $1 \mathrm{C}$, IFh (5'-TCCCTTCTCTCATGACTCTCATCTCTACGC-3', 22 nucleotides upstream to exon 2) and IRk (5'-ACACCAACAGGCAGCGGCCTAGAAGCATGG-3' ${ }^{\prime}$, antisense sequence, 14 nucleotides downstream to exon 2) for exon 2, and IFg (5'-GTCGGAGAGTACCTCTAGTATTCTGTAAGTTC$3^{\prime}, 57$ nucleotides upstream to exon 3) and IRc for exon 3. The PCR products were cloned into the pCRII-TOPO vectors, and the DNA sequences were determined. Multiple clones from 2 batches of PCR products were sequenced to distinguish any PCR error from actual sequence polymorphism.

\section{PCR-restriction fragment length polymorphism (RFLP) analysis}

The $505 \mathrm{G}>\mathrm{A}$ mutation identified in the $I G n T C$ gene destroys a $B s t \mathrm{NI}$ recognition sequence (CCWGG), and thus a PCR-RFLP analysis was 
developed to detect the mutation. The 606-base pair (bp) fragment encompassing the 505-nucleotide position of the IGnTC gene was PCR amplified using genomic DNA as a template and primers ICF9 (5'AGAAGAGGCTGCATTCCC-3', nucleotides 270-287 of IGnTC) and ICR11 (5'-GATCAATGGCCCTTTGGTCACG-3', complementary of nucleotides $835-856$ of $I G n T C$ ). The PCR program included 5 minutes at $94^{\circ} \mathrm{C}$ followed by 30 cycles of 30 seconds at $94^{\circ} \mathrm{C}, 30$ seconds at $58^{\circ} \mathrm{C}$, and 1 minute at $72^{\circ} \mathrm{C}$. The PCR products were subjected to digestion by $B s t \mathrm{NI}$ restriction endonuclease and were then analyzed by agarose gel electrophoresis. The PCR product amplified from the wild-type IGnTC allele was cleaved into 233-, 225-, 101-, and 47-bp fragments, whereas that from the mutant IGnTC allele with the $505 \mathrm{G}>$ A mutation $(I G n T C * 505 A)$ yielded 280-, 225-, and 101-bp fragments.

A PCR-RFLP analysis for $F s p$ I was designed to detect the $683 \mathrm{G}>\mathrm{A}$ mutation identified in the IGnTC gene. The 683-nucleotide position of the IGnTC gene was sampled by PCR amplification using primers ICFm55 (5'-AGGGGTGCTGCCTCCTGACCATGCAATTGCGC-3', nucleotides 651-682 of IGnTC) and ICR56 (5'-GATGGTCAGCTGATGTGGAGGTGAAGT-3', complementary of nucleotides 754-780 of IGnTC). In the ICFm55 primer, 2 nucleotides (boldfaced) were changed from AA to GC, so that an $F s p$ I recognition sequence (TGCGCA) would be produced when a mutant $I G n T C$ allele with the $683 \mathrm{G}>$ A mutation $(I G n T C * 683 A)$ was used as a template to produce the PCR product. The PCR program included 5 minutes at $94^{\circ} \mathrm{C}$ followed by 30 cycles of 30 seconds at $94^{\circ} \mathrm{C}, 30$ seconds at $65^{\circ} \mathrm{C}$, and 30 seconds at $72^{\circ} \mathrm{C}$. After digestion by $F s p I$ restriction endonuclease, the 130-bp PCR product amplified from the IGnTC*683A allele was cleaved into 100- and 30-bp fragments, whereas that from the wild-type $I G n T C$ allele was resistant to digestion.

\section{Functional analyses of the enzymes encoded from the IGnT cDNAs}

The cDNA fragment encompassing the region of nucleotides 79 to 1206 , which encodes the amino acid residues 27 to 402, of the IGnTA gene was prepared by RT-PCR and primers IAF ( 5 '-aattggcccagccggccGAGTTATGGGAGAATAAACG-3') and IRx (5'-ttaagggcccAAAATACCAGCTGGGTTGTATCGCAG- $3^{\prime}$, antisense sequence). Primers ICF (5'-aattggcccagccggccCAATTGAGCCCGCCAAAAAGTTATGAG- $3^{\prime}$ ) and IRx were used to prepare the cDNA fragment encompassing the region of nucleotides 76 to 1206, which encodes the amino acid residues 26 to 402, of the IGnTC gene. The primers contained SfiI and ApaI recognition sequences (underlined) at the $5^{\prime}$ ends, respectively. Total RNA prepared from peripheral blood cells of an individual with the common I phenotype and an individual with the adult i phenotype (W-3), who is a heterozygote with the IGnTB*1043A/ $I G n T B * 1148 A$ genotype, served as templates. The amplified cDNA fragments were cloned into SfiI and ApaI sites of the mammalian expression vector pSecTaq2A (Invitrogen), which is designed for the secretion of the expressed protein by the $\mathrm{N}$-terminal secretion signal from the $\mathrm{V}-\mathrm{J} 2-\mathrm{C}$ region of the mouse immunoglobulin $\kappa$ chain. Vectors bearing wild-type IGnTA and IGnTC, and mutant IGnTA*1049A, IGnTA*1154A, IGnTC*1049A, and $I G n T C * 1154 A$ cDNAs, were selected and sequence-confirmed. Construction of the vectors bearing wild-type IGnTB cDNA has been described previously. 23

The expression vectors bearing mutant $I G n T C * 505 A$ and $I G n T C * 683 A$ cDNAs were constructed as follows. DNA fragments were obtained from PCR amplification using ICF and ICR6 as primers and genomic DNA from the adult i individual (1110X3), who is a heterozygote with the IGnTC*505A/ $I G n T C * 683 A$ genotype, as a template, and were digested by SalI restriction endonuclease. The SalI-cleaved fragments, encompassing the region of nucleotides 76 to 822 of IGnTC, were selected and ligated with the fragments encompassing the region of nucleotides 823 to 1206 obtained by Sall digestion of the wild-type IGnTC cDNA product, amplified by the ICF and IRx primers mentioned in the preceding paragraph. The ligated fragment was cloned into $S$ fiI and $A p a \mathrm{I}$ sites of the pSecTaq2A vector, and vectors bearing the respective IGnTC*505A and IGnTC*683A cDNAs were selected and sequence-confirmed.

The constructed and the mock pSecTaq2A plasmids were prepared using the EndoFree Plasmid Kit (Qiagen) for transfection. Expression of the constructed and mock plasmids in COS-7 cells, and the subsequent GlcNAcT assay, were performed as described previously. ${ }^{23}$

\section{Preparation of the RNA samples from reticulocytes and lens-epithelium cells}

Pure reticulocytes were isolated from whole blood through positive isolation of cells expressing the transferrin receptor (CD71) by immunomagnetic separation. ${ }^{28}$ Eight milliliters of venous blood from a healthy adult was drawn in a tube containing anticoagulant, and, after centrifugation at $2300 \mathrm{~g}$ for 15 minutes, white blood cells (the buffy coat layer) were removed. Dynabeads CD71 (Dynal Biotech, Lake Success, NY), which are immunomagnetic beads coated with monoclonal antibody against human transferrin receptor, were then used to isolate the reticulocytes, following the protocol provided by the manufacturer.

Four lenses were obtained from 4 individuals who had undergone eye surgery. Total RNA samples from the isolated reticulocytes and from the lens-epithelium cells were purified using the RNeasy Mini Kit (Qiagen).

\section{Results}

\section{IGnTA, IGnTB, and IGnTC are expressed from the human Ilocus}

The human IGnT gene is located on chromosome $6 \mathrm{p} 24$. The coding sequence for the gene is divided into 3 exon regions, with respective coding nucleotides of 919,93 , and $191 \mathrm{bp} .{ }^{29}$ Basic Local Alignment Search Tool (BLAST) analysis of the gene databases at the National Center for Biotechnology Information (National Institutes of Health, Bethesda, MD) with the exon 1 coding sequence of the $I G n T$ gene revealed 2 novel open reading frames with significant homology. The relative positions of these 2 novel regions to exons 1,2 , and 3 of the $I G n T$ gene were known from BAC clone RP11-360019 on human chromosome 6 (GenBank accession no. AL139039) (Figure 1). In this paper, the 2 novel open reading frames are designated $1 \mathrm{~A}$ and $1 \mathrm{C}$, with the original exon 1 region of the previously reported $I G n T$ gene designated as $1 \mathrm{~B}$.

From the 5'-RACE and RT-PCR analyses, it was demonstrated that the homologous $1 \mathrm{~A}$ and $1 \mathrm{C}$ regions are transcribed and also processed to link with the exon 2 and exon 3 regions, as for exon 1B (Figure 1). Both of the intron sequences at the exon-intron junctions conform to the GT-AG consensus (data not shown). Thus, 3 transcripts with different exon 1, but identical exons 2 and 3, coding regions are expressed from the human $I$ locus. The 3 transcripts are designated IGnTA, IGnTB, and IGnTC. Exon $1 \mathrm{~A}$ and exon $1 \mathrm{C}$ consist of 925 coding nucleotides. The $I G n T B$ is the original $I G n T$ gene reported by Bierhuizen et al. ${ }^{27}$

The 5' - and 3'-RACE, performed on cDNA derived from human prostate to establish the $I G n T$ cDNA structures, yielded

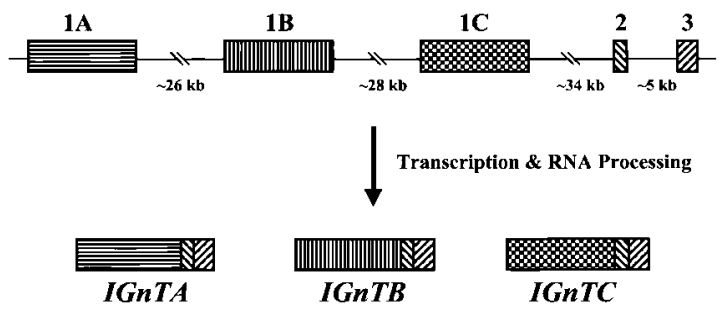

Figure 1. Schematic representation of the organization of the human I locus and the structures of the expressed IGnT gene. Three IGnT transcripts, IGnTA $I G n T B$, and IGnTC, which have different exon 1 but identical exon 2 and exon 3 coding regions, are expressed from the human / locus. The coding nucleotides of exons $1 \mathrm{~A}, 1 \mathrm{~B}$, and $1 \mathrm{C}$ have 925,919 , and $925 \mathrm{bp}$, respectively, and the common exon 2 and exon 3 have respective coding nucleotides of 93 and $191 \mathrm{bp}$. 
products of different sizes, indicating the possible existence of different transcript structures for the $3 I G n T \mathrm{~s}$. The full cDNA structures of the IGnTA, IGnTB, and IGnTC transcripts obtained from prostate cDNA (supplemental data on the Blood website [see the Supplemental Figure link at the top of the online article]; GenBank/EBI DataBank accession numbers AF458024, AF458025, and AF458026, respectively) have open reading frames of 1209 , 1203, and $1209 \mathrm{bp}$, respectively, which predict respective protein products of 402, 400, and 402 amino acid residues (Figure 2). The protein products predicted from the novel IGnTA and IGnTC cDNAs have potential hydrophobic transmembrane segments at $\mathrm{N}$-terminals, as does the IGnTB-encoded $\beta 6 \mathrm{GlcNAcT}$, and $73 \%$ sequence identity with the IGnTB $\beta 6$ GlcNAcT is revealed for both. Conservation of 9 Cys residues and $66 \%$ overall sequence identities are demonstrated for the 3 IGnT proteins.

Expression profiles for the IGnTA, IGnTB, and IGnTC transcripts for different human tissues were analyzed using RT-PCR. As shown in Figure 3, wide distributions were noted for the $3 I G n T$ transcripts in the human tissues analyzed, except that IGnTA was not detected in the RNA samples from the brain, and it appeared that different $I G n T$ forms are expressed differentially in quantity in various human tissues, although the RT-PCR analysis was not quantitative.

The protein products encoded from the novel IGnTA and IGnTC cDNAs were expressed in mammalian cells (COS-7), and their potential GlcNAcT activity was examined and compared with that of the I $\beta 6 \mathrm{GlcNAcT}$ encoded from the IGnTB cDNA. The amounts of GlcNAc transferred to the acceptor substrate (LS-tetrasaccharide c [NeuNAc $\alpha 2-6 \mathrm{Gal} \beta 1-4 \mathrm{GlcNAc} \beta 1-3 \mathrm{Gal} \beta 1-4 \mathrm{Glc}])$ from the donor substrate (UDP-GlcNAc) by the medium concentrates harvested from the cells transfected with respective expression vectors were measured. GlcNAcT activity was demonstrated in the enzyme function assay for the expressed IGnTA and IGnTC protein products, as was the IGnTB $\beta 6$ GlcNAcT (Figure 4); the IGnTC enzyme appeared to have the highest GlcNAc-transferring activity. It has been demonstrated that the predominant activity of the enzyme encoded from the $I G n T B$ transcript is I-branching $\beta 6 \mathrm{Glc}$ NAcT activity. ${ }^{27,30}$ Compared with the IGnTB $\beta 6$ GlcNAcT, comparable or even higher GlcNAcT activity was demonstrated for the IGnTA and IGnTC enzymes with the same acceptor substrate,

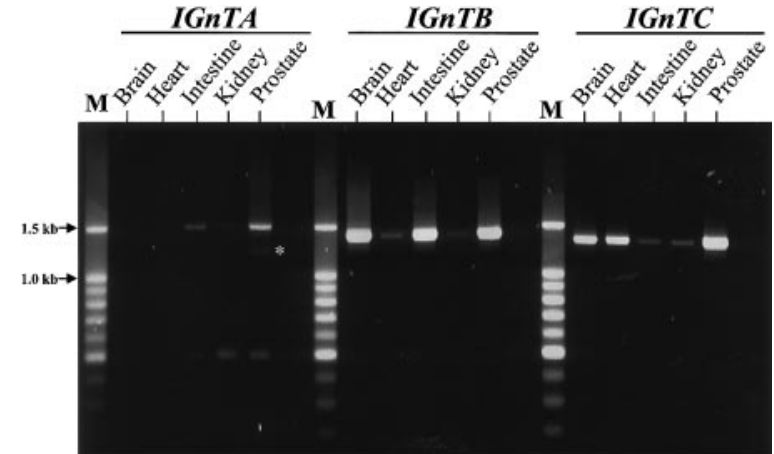

Figure 3. Expression profiles for the IGnT gene from various human tissues. Poly $\mathrm{A}^{+}$RNA samples from the human brain (cerebellum), heart, small intestine, kidney, and prostate were primed using oligo-dT primer to synthesize the first-strand cDNA. Then, PCR using gene-specific forward primer and common reverse primer was performed, as described in "Patients, materials, and methods." The RT-PCR products were analyzed using $1.5 \%$ agarose gel electrophoresis. The expected sizes of the products from the IGnTA, IGnTB, and IGnTC transcripts were 1486, 1391, and $1326 \mathrm{bp}$, respectively. From RT-PCR for the IGnTA, an additional product approximately 500 bp in size, consisting of a shorter exon 1 A region conjoining with exon 2-3 regions, was observed. This smaller product is believed to result from alternative splicing and does not have a correct reading frame relative to the exon 2-3 coding sequence. The faint bands (indicated by an asterisk) below those of the IGnTA 1486-bp fragment were the hybrid complex of the IGnTA 1486-bp and the alternatively spliced 500-bp products.

which has been shown to be a good acceptor substrate for the I $\beta 6$ GlcNAcT activity assay. Nevertheless, further carbohydrate structure analysis of the products generated from IGnTA and IGnTC activity is required to characterize the GlcNAcT activity and demonstrate the I $\beta 6$ GlcNAcT nature of the IGnTA and IGnTC enzymes.

\section{Wild-type IGnTA and IGnTB, but mutant IGnTC, are present in the adult $i$ whites without congenital cataracts}

The exon 1A, 1B, 1C, 2, and 3 regions of the $I G n T$ gene of the individual with the adult $\mathrm{i}$ phenotype (JCC) were amplified by PCR, cloned, and sequence-analyzed. The coding sequences for these exon regions and the adjacent splice sites were inspected, and the wild-type sequences of the exon $1 \mathrm{~A}, 1 \mathrm{~B}, 2$, and 3 regions in this

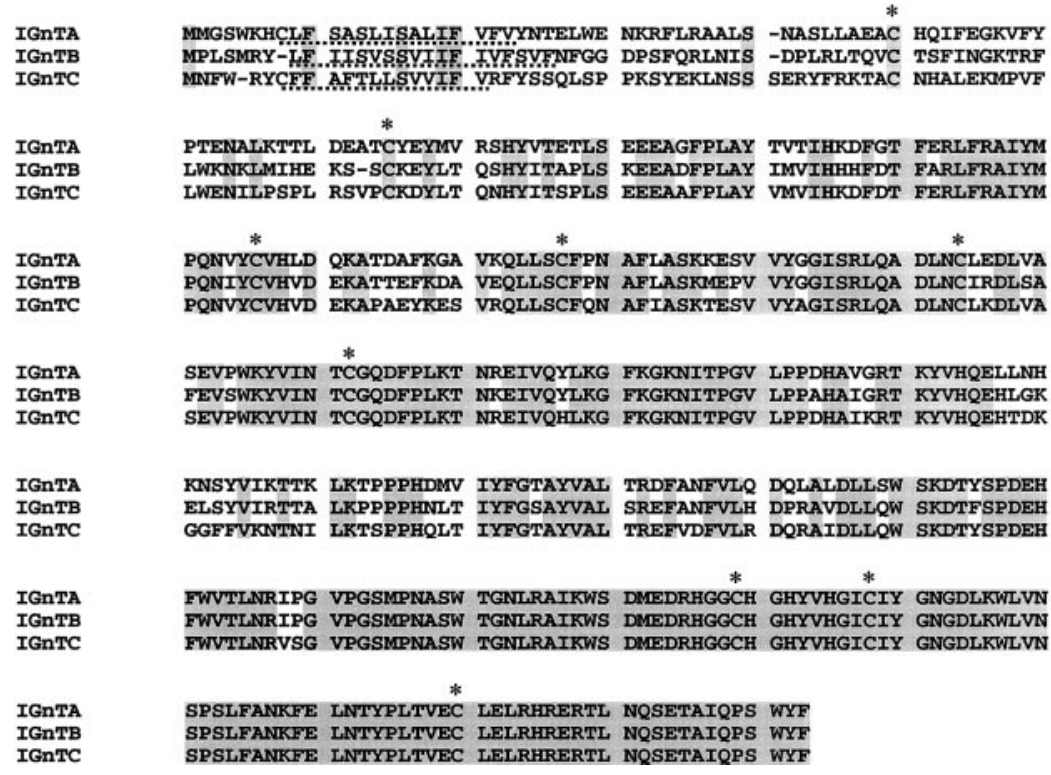

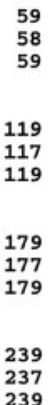

237
239

299

297

359

357 359

Figure 2. Amino acid sequences deduced from the 402 400 402 Cys residues are marked by asterisks. Dashed lines underline the hydrophobic segments at the $\mathrm{N}$-terminals. 


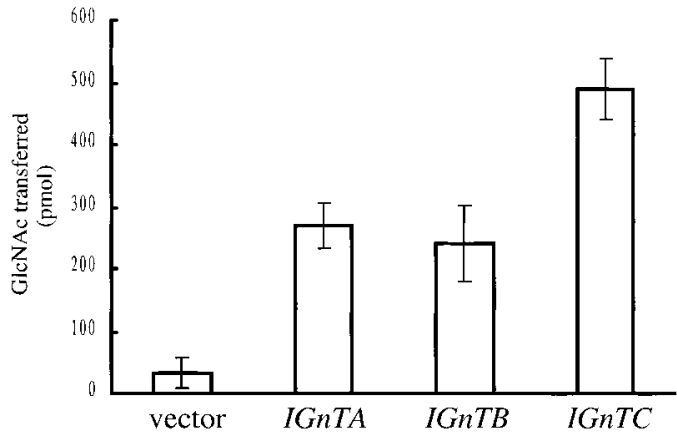

Figure 4. The GIcNAcT activities of the enzymes encoded from the IGnTA $I G n T B$, and IGnTC cDNAs. The protein products encoded from the IGnTA, IGnTB and IGnTC cDNAs were expressed in mammalian cells (COS-7). The amounts of GlcNAc transferred to the acceptor substrate (LS-tetrasacchride c [NeuNAca2-6Galb14GlcNAcb1-3Galb1-4Glc]) from the donor substrate (UDP-GlcNAc) by the medium concentrates harvested from the cells transfected with respective expression vectors were measured as has been described previously. ${ }^{23}$ The results of the average and standard deviation (indicated by error bars) of 4 tests are diagrammatically represented. Endogenous transfer of GlcNAc in the absence of acceptor substrate was corrected for each test. The amounts of the transferred GlcNAc in the vector control, pSecTaq2A, indicate the background levels of the assay, which are believed to result from the addition of the acceptor substrate.

adult $\mathrm{i}$ white were demonstrated, with a $\mathrm{G}>\mathrm{A}$ substitution at the 505 nucleotide position $(505 \mathrm{G}>\mathrm{A})$ of exon $1 \mathrm{C}$ identified. All 4 of the analyzed clones bearing the exon $1 \mathrm{C}$ region possessed the $505 \mathrm{G}>$ A mutation, suggesting that the individual was homozygous for the $505 \mathrm{G}>\mathrm{A}$ mutation.

A PCR-RFLP for BstNI was developed (as described in "Patients, materials, and methods") and used to detect the 505G $>$ A mutation for the 6 adult $i$ whites. It was demonstrated that 5 of the 6 samples (JCC, 97013, 987U1, 944C2, and 1110Y2) were homozygous for the $505 \mathrm{G}>\mathrm{A}$ mutation in the IGnTC gene, whereas the remaining individual, 1110X3, harbored one mutant $I G n T C$ allele with the $505 \mathrm{G}>\mathrm{A}$ mutation (data not shown).

The IGnT exon $1 \mathrm{~A}, 1 \mathrm{~B}, 1 \mathrm{C}, 2$, and 3 regions of the $1110 \mathrm{X} 3$ individual were PCR-amplified, cloned, and analyzed. Wild-type sequences of the exon $1 \mathrm{~A}, 1 \mathrm{~B}, 2$, and 3 regions were also demonstrated for this white $\mathrm{i}$ adult and, in addition to the IGnTC allele with the $505 \mathrm{G}>\mathrm{A}$ mutation, another allele with the $683 \mathrm{G}>\mathrm{A}$ mutation in the exon $1 \mathrm{C}$ region was identified. The PCR-RFLP for FspI, which was developed to detect the $683 \mathrm{G}>\mathrm{A}$ mutation, proved the heterozygosity of the $683 \mathrm{G}>\mathrm{A}$ mutation in this individual.

The $505 \mathrm{G}>\mathrm{A}$ and $683 \mathrm{G}>\mathrm{A}$ mutations in the $I G n T C$ predict the amino acid alterations of Ala169Thr and Arg228Gln, respectively.
The 2 mutated IGnTC alleles are designated IGnTC*505A and $I G n T C * 683 A$ (Figure 5A). These results suggest the presence of wild-type IGnTA and IGnTB in these adult i whites and demonstrate their carriage of double-dose mutant IGnTC alleles. Five of the 6 whites were homozygous for the $I G n T C * 505 A$ allele, and one was a heterozygote with the IGnTC*505A/IGnTC*683A genotype.

\section{The IGnTC ${ }^{\star} 505 A$ and $I G n T C{ }^{\star} 683 A$ alleles are uncommon in the general white population}

The incidence of the IGnTC*505A and $I G n T C * 683 A$ alleles in the white population was evaluated using PCR-RFLP analyses. Genomic DNAs obtained from 51 randomly selected whites were screened. It was determined that one individual possessed one $I G n T C * 505 \mathrm{~A}$ allele and that none had the $683 \mathrm{G}>\mathrm{A}$ mutation in the IGnTC (data not shown). These results indicate that the $2 \mathrm{G}>\mathrm{A}$ mutations in the IGnTC gene are uncommon in this ethnic group. The low incidence of the $I G n T C * 505 A$ and $I G n T C * 683 A$ alleles agrees with the observed rarity of the adult i phenotype.

\section{All 3 IGnT forms of the adult i Taiwanese individuals with congenital cataracts were mutated}

Previously, the IGnTB genes of the 5 Taiwanese with the adult i phenotype were analyzed, and 3 different molecular changes were identified: missense mutations of $1043 \mathrm{G}>\mathrm{A}$ and $1148 \mathrm{G}>\mathrm{A}$ and gene deletion of the exon $1 \mathrm{~B}$, exon 2 , and exon 3 regions. ${ }^{23}$ The $1043 \mathrm{G}>\mathrm{A}$ and $1148 \mathrm{G}>\mathrm{A}$ mutations predict the amino acid alterations of Gly348Glu and Arg383His, respectively, in the encoded IGnTB $\beta 6$ GlcNAcT enzyme. Pedigree analysis and enzyme function assay further demonstrated the association between these molecular changes and the adult i phenotype.

Because 2 new exon regions, $1 \mathrm{~A}$ and $1 \mathrm{C}$, which contribute to produce 2 novel $I G n T$ forms, were identified in the present study, the samples from the adult i Taiwanese were reanalyzed. The exon $1 \mathrm{~A}$ and $1 \mathrm{C}$ regions of subject $\mathrm{W}-3$, demonstrated as heterozygous for the $I G n T B * 1043 A / I G n T B * 1148 A$ genotype, were cloned and sequence-analyzed. No abnormalities were found in the exon $1 \mathrm{~A}$ and $1 \mathrm{C}$ regions. The result indicates that, except for the 2 previously identified mutations, no additional mutation is present in the exon $1 \mathrm{~A}$ and $1 \mathrm{C}$ regions of the adult $\mathrm{i}$ Taiwanese subject, W-3.

The 2 previously identified missense mutations, $1043 \mathrm{G}>\mathrm{A}$ and $1148 \mathrm{G}>\mathrm{A}$, are located in the exon 3 region; however, the exon 3 for the IGnTB is also common to the IGnTA and IGnTC. Thus, the $\mathrm{G}>\mathrm{A}$ change in the $I G n T B^{*} 1043 A$ cDNA also yields the
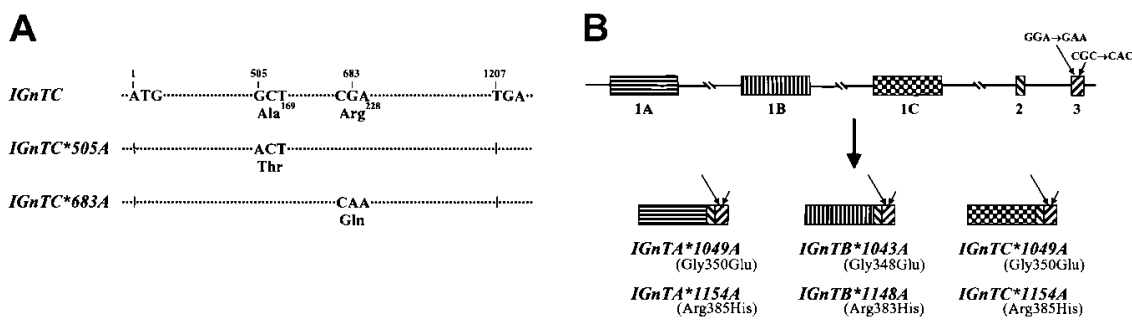

Figure 5. Mutations identified in the IGnT gene of adult i individuals. (A) Mutations identified in the IGnTC gene of the adult i whites. IGnTC indicates the wild-type coding sequence of the IGnTC gene. ATG and TGA correspond to the respective translation start and stop codons. The mutant alleles identified in white subjects with the adult $i$ phenotype, designated $I G n T C{ }^{*} 505 A$ and $I G n T C^{*} 683 A$, possess $505 G>A$ and $683 G>A$ nucleotide substitutions, respectively, which predict the respective amino acid alterations of Ala169Thr and Arg228Gln. (B) Schematic representation of the mutations identified in the IGnT gene of adult i Taiwanese with congenital cataracts. The $2 \mathrm{G}>\mathrm{A}$ changes, identified, as reported previously, at the 1043 and 1148 nucleotide positions of the IGnTB gene, locate in the common exon 3 region of the $3 / G n T$ forms, and, thus, the 2 mutations also contribute to form the mutant IGnTA*1049A and IGnTC*1049A as well as IGnTA*1154A and IGnTC*1154A, respectively. Exons $1 \mathrm{~A}$ and $1 \mathrm{C}$ possess 6 more coding nucleotides than the exon 1B does, and thus the nucleotide numbers of the same $\mathrm{G}>\mathrm{A}$ changes in the IGnTA and IGnTC transcripts are different from those in the IGnTB transcripts. The Gly-to-Glu and Arg-to-His alterations resulting from the mutations in the encoded enzyme products are shown. 
IGnTA*1049A and IGnTC*1049A transcripts, and the $\mathrm{G}>\mathrm{A}$ change in the $I G n T B * 1148 A$ cDNA also produces the $I G n T A * 1154 A$ and $I G n T C * 1154 A$ transcripts (Figure 5B). The IGnTA*1049A, $I G n T A * 1154 A, I G n T C * 1049 A$, and IGnTC*1154A, as well as the $I G n T B * 1043 A$ and $I G n T B * 1148 A$, transcript structures were demonstrated in the blood cell RNA sample from i adult W-3 with the use of RT-PCR.

For $\mathrm{i}$ adult $\mathrm{C}-3$, it was demonstrated previously that the exon 1B, exon 2, and exon 3 regions were deleted. PCR amplifications of the genomic DNA sample from $\mathrm{C}-3$ were performed to inspect the exon $1 \mathrm{~A}$ and $1 \mathrm{C}$ regions. As expected, the exon $1 \mathrm{C}$ region was absent, but the exon 1A region appeared to be intact in the C-3 individual (data not shown). However, because of the deletion of the exon 2 and exon 3 regions, it is believed that the complete $I G n T A$ transcript, as well as the IGnTB and IGnTC transcripts, are lacking in i adult $\mathrm{C}-3$.

Thus, all $3 I G n T$ forms are mutated in these adult i Taiwanese. Subjects W-3 and W-5 are heterozygotes with IGnTA*1049A/ $I G n T A * 1154 A, I G n T B * 1043 A / I G n T B * 1148 A$, and IGnTC*1049A/ IGnTC* $1154 A$ genotypes, and S-6 and S-7 are homozygous for the $I G n T A * 1049 A, I G n T B * 1043 A$, and $I G n T C * 1049 A$ alleles, whereas the $3 I G n T$ s are deleted completely or partially in C-3.

\section{Activity of the enzymes encoded from the mutant IGnT genes}

The effects of the Ala169Thr and Arg228Gln changes, resulting from the $505 \mathrm{G}>\mathrm{A}$ and $683 \mathrm{G}>\mathrm{A}$ mutations in the IGnTC genes, respectively, on the GlcNAc-transferring activity were examined using the enzyme function assay. Although residual activity was observed, the enzyme activity of the products encoded from the $I G n T C * 505 A$ and $I G n T C * 683 A$ cDNAs was dramatically reduced when compared with that of the wild-type IGnTC-encoded enzyme (Table 1).

In our previous report, the Gly348Glu and Arg383His alterations, predicted from $1043 \mathrm{G}>\mathrm{A}$ and $1148 \mathrm{G}>\mathrm{A}$, respectively, totally eliminated the original enzyme activity of the IGnTBencoded $\beta 6$ GlcNAcT. The $2 \mathrm{G}>\mathrm{A}$ changes in the mutant IGnTA and IGnTC genes also led to the Gly350Glu and Arg385His alterations, respectively, in their corresponding enzymes (Figure $5 \mathrm{~B})$. The activities of the enzymes encoded from the $I G n T A * 1049 A$ and $I G n T C * 1049 A$ and the IGnTA*1154A and IGnTC*1154A transcripts were inspected. The Gly350Glu and Arg385His changes in the C-terminal segments of the IGnTA- and IGnTC-encoded GlcNAcTs effectively eliminated the original GlcNAc-transferring activity, similar to the effects of the Gly348Glu and Arg383His changes on IGnTB $\beta 6$ GlcNAcT (Table 1).

Table 1. GIcNAcT activities of the enzymes encoded from the mutant IGnTA and IGnTC cDNAs

\begin{tabular}{lc}
\hline & GlcNAc transferred, pmol \\
\hline Vector & $26.7 \pm 8.5$ \\
IGnTA & $143.0 \pm 5.4$ \\
IGnTA*1049A & $23.2 \pm 4.9$ \\
IGnTA*1154A & $24.3 \pm 3.9$ \\
Vector & $7.2 \pm 2.3$ \\
IGnTC & $210.9 \pm 63.5$ \\
IGnTC*505A & $24.5 \pm 5.1$ \\
IGnTC*683A & $15.6 \pm 3.0$ \\
IGnTC*1049A & $12.6 \pm 2.1$ \\
IGnTC*1154A & $4.7 \pm 6.0$ \\
\hline
\end{tabular}

Results are the averages and standard deviations of 4 tests. Endogenous transfer of GlcNAc in the absence of acceptor substrate was corrected for each test.
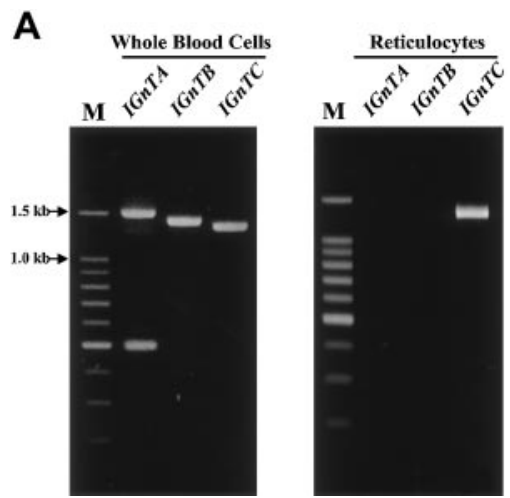

B

Figure 6. RT-PCR analyses of IGnT gene expression in the RNA samples purified from whole blood cells and reticulocytes and from lens-epithelium cells. Pure reticulocytes were isolated from whole blood through positive isolation of cells expressing the transferrin receptor (CD71) by immunomagnetic separation, as described in "Patients, materials, and methods." Total RNA from whole blood cells (the buffy coat layer) and reticulocytes (A) and lens-epithelium cells (B) was prepared, and RT-PCR analysis for the IGnTA, IGnTB, and IGnTC transcripts was conducted as described in "Patients, materials, and methods" and the legend of Figure 3

\section{IGnTC is the only one of 3 IGnT transcripts expressed in reticulocytes, whereas only the IGnTB transcript is expressed in lens-epithelium cells}

The expression of the $3 I G n T$ transcripts in reticulocytes, the RBC precursors, was examined using RT-PCR. Pure reticulocytes were isolated from white cell-depleted whole blood cells through positive selection of CD71 marker-expressing cells by immunomagnetic separation (as described in "Patients, materials, and methods"). The RT-PCR analyses revealed that the IGnTA, IGnTB, and $I G n T C$ transcripts were detected in the RNA purified from whole blood cells, whereas only the IGnTC transcript was detected in the RNA purified from reticulocytes (Figure 6A), indicating that the $I G n T C$ gene is the only one of the $3 I G n T$ forms expressed in reticulocytes.

By contrast, RT-PCR analysis demonstrated only the expression of the IGnTB transcript in the RNA purified from human lensepithelium cells (Figure 6B).

\section{Discussion}

The present study has demonstrated not only that the human $I$ locus expresses the previously reported $I G n T$ transcript, but also that 3 $I G n T$ forms (IGnTA, IGnTB, and IGnTC), which have different exon 1 but identical exon 2 and exon 3 coding regions, are expressed from the human $I$ locus. A similar molecular mechanism has been observed in the human UDP-glucuronosyltransferase UGT1A locus, which contains at least 12 promoters/first exons, which can be spliced and joined with common exons 2 through 5, leading to the formation of 12 different isoforms. ${ }^{31}$ The enzyme encoded from the $I G n T B$ gene has been shown to exhibit the I- $\beta 6$ GlcNAcT activity. ${ }^{27,31}$ When an acceptor substrate that mimics the structure of the i precursor was used in the enzyme function assay, similar or even higher GlcNAcT activity was demonstrated for the enzymes encoded from the novel IGnTA and IGnTC in comparison with IGnTB $\beta 6$ GlcNAcT, suggesting that the IGnTA and IGnTC enzymes also have I- $\beta 6$ GlcNAcT activity. Nevertheless, further enzyme characterizations for the novel GlcNAcTs 
Table 2. I genotypes and IGnT enzyme activities of the i adults without and with congenital cataracts

\begin{tabular}{|c|c|c|c|c|c|c|c|}
\hline \multirow[b]{2}{*}{ i Adult group } & \multirow[b]{2}{*}{ Patient no. } & \multicolumn{3}{|c|}{ Igenotype } & \multicolumn{3}{|c|}{ Enzyme activity } \\
\hline & & IGnTA & IGnTB & IGnTC & IGnTA & IGnTB & IGnTC \\
\hline \multirow[t]{6}{*}{ Without congenital cataracts } & $\mathrm{JCC}$ & WT & WT & $505 / 505$ & $\sqrt{ }$ & $\sqrt{ }$ & $x$ \\
\hline & 97013 & ND & ND & $505 / 505$ & ND & ND & $x$ \\
\hline & $987 \mathrm{UI}$ & ND & ND & $505 / 505$ & ND & ND & $x$ \\
\hline & $944 C 2$ & ND & ND & $505 / 505$ & ND & ND & $x$ \\
\hline & $1110 Y 2$ & ND & ND & $505 / 505$ & ND & ND & $x$ \\
\hline & $1110 \times 3$ & WT & WT & $505 / 683$ & $\sqrt{ }$ & $\sqrt{ }$ & $x$ \\
\hline \multirow[t]{5}{*}{ With congenital cataracts } & W-3 & $1049 / 1154$ & $1043 / 1148$ & $1049 / 1154$ & $x$ & $x$ & $x$ \\
\hline & W-5 & $1049 / 1154$ & $1043 / 1148$ & $1049 / 1154$ & $x$ & $x$ & $x$ \\
\hline & S-6 & $1049 / 1049$ & $1043 / 1043$ & $1049 / 1049$ & $x$ & $x$ & $x$ \\
\hline & S-7 & $1049 / 1049$ & $1043 / 1043$ & $1049 / 1049$ & $x$ & $x$ & $x$ \\
\hline & C-3 & Deletion & Deletion & Deletion & $x$ & $x$ & $x$ \\
\hline
\end{tabular}

The numbers $505,683,1043,1049,1148$, and 1154 represent the $\mathrm{G}>\mathrm{A}$ change at respective nucleotide positions.

WT indicates wild type; ND, not determined; $\sqrt{ }$, normal enzymatic activity, $X$, decreased enzymatic activity.

encoded from the IGnTA and IGnTC forms are required to demonstrate their I $\beta 6 \mathrm{GlcNAcT}$ activity. Whether these 2 novel enzymes possess any other glycosyltransferase specificity other than the I- $\beta 6$ GlcNAcT activity is also worthy of further investigation.

It has been demonstrated that the expression of the I antigen is developmentally regulated and altered during the process of oncogenesis. At present, it has been determined that the human $I$ locus expresses 3 different $I G n T$ forms and that the $3 I G n T$ s are expressed differentially in different tissues and cells. The 5'-RACE analyses revealed that the IGnTA, IGnTB, and IGnTC cDNAs did not have a common $5^{\prime}$ region, indicating that transcription of the 3 $I G n T$ forms may be determined by different DNA regulatory regions or by different regulatory mechanisms. Thus, when attempting to understand the expression of the I antigen in different tissues and cells and the mechanisms for the appearance and disappearance of the I antigen during developmental and oncogenetic processes, one should consider the functional roles of the 3 individual IGnT forms. Further, it may be of interest to examine the expression profiles for the various $I G n T$ transcripts during cell-differentiation stages and to elucidate the regulatory mechanisms for each of the 3 $I G n T$ forms to understand how the differential regulation of the $I G n T$ s may be manipulated.

Although detailed enzyme characterization of the newly revealed $I$ gene glycosyltransferase products remains indefinite and awaits further elucidation, this molecular genetic analysis of the human $I$ locus provides insight into the questions derived from investigation of the adult $i$ phenotype, including the partial association of the adult i phenotype with congenital cataracts.

It has long been noted that the human $I$ locus is correlated with congenital cataracts. Although a significant association of the adult i phenotype with congenital cataracts has been demonstrated for Asians, it is not as pronounced for the white population. Thus, it has been suggested that the association results from a close linkage of 2 independent I- and cataract-related genes, rather than from a pleiotropic effect of the gene responsible for the adult i phenotype on the development of cataracts. It was further surmised that the association may be a result of deletion of a chromosomal region that encompasses the 2 independent I- and cataract-related genes. Nevertheless, we demonstrated in our previous study that nucleotide substitutions were the molecular origins of the adult $i$ phenotype in Taiwanese with congenital cataracts, and this result highly suggests that the association may result from the pleiotropic effect of the same mutant gene.

The IGnT genotypes for the 5 adult i Taiwanese and 6 adult i whites, with and without congenital cataracts, respectively, to- gether with their IGnT enzyme activities as inferred from the enzyme function assays, are presented in Table 2 . The adult $i$ whites without congenital cataracts have wild-type IGnTA and IGnTB gene forms but possess a double dose of the mutant IGnTC genes, which encode enzyme product with markedly low GlcNAcT activity, whereas all $3 I G n T$ forms are mutated and all of the activities of the encoded GlcNAcTs are abolished in the adult $i$ Taiwanese with congenital cataracts.

The first conclusion that can be drawn from these results is that the exact gene form responsible for the expression of the blood group I antigen on RBCs should be assigned to the IGnTC, not the $I G n T B$, as previously described. ${ }^{23}$ This conclusion is further supported by the observation that IGnTC is the only one of the 3 $I G n T$ forms expressed in reticulocytes, the RBC precursors.

Second, the molecular genetics of the human $I$ locus support the proposition that more than one I-branching enzyme exists. The proposition that different I-branching enzymes may be responsible for I-antigen synthesis in different tissues is based on the presence of normal quantities of I antigen in the saliva, milk, and plasma of $i$ adults. $^{4,32,33}$ The $3 I G n T$ forms display differential expression patterns in different tissues and cells, as illustrated in Figures 3 and 6. We have analyzed the expression of the IGnT gene in human mammary and salivary glands by RT-PCR, with the results demonstrating that the IGnTB transcript is the predominant form of the $3 I G n T$ transcripts expressed in these 2 tissues (data not shown). If the IGnTB $\beta 6 \mathrm{GlcNAcT}$ is responsible for I-antigen formation in these 2 tissues, normal expression of this antigen in the milk and saliva of the $\mathrm{i}$ adults with defective IGnTC but normal IGnTB would be expected.

The most significant conclusion that can be drawn from these results is that a molecular genetic mechanism accounting for the partial association of the adult $i$ phenotype with congenital cataracts is suggested. Mutation events that occur in the specific exon 1 region of the $I G n T$ gene may lead to a defect in one form of IGnT enzyme activity in certain cell types that express the specific $I G n T$ gene form, whereas those that occur in the common exon 2-3 region may result in elimination of the activity of all 3 of the IGnT enzymes. This is precisely what was observed for the 2 adult $i$ groups with or without congenital cataracts, and the molecular events observed for the 2 groups are consistent. The human lens cells are differentiated from the surface of a single layer of epithelium cells. RT-PCR analysis detected only IGnTC transcript in the RNA purified from reticulocytes, however, with only the $I G n T B$ expressed in the human lens-epithelium cells (Figure 6). A defect in IGnTC gene function leads to the absence of I antigen in 
RBCs, whereas congenital cataracts occur in those i adults in whom all 3 IGnT-enzyme functions are defective, but not in analogs in which only the IGnTC form is defective (Table 2).

The molecular genetics of the human $I$ locus revealed in the present study provide a new view of the formation and expression of the I antigen. The architecture of the I-gene structures and the results obtained from the molecular analysis of the $i$ adults answer many questions about the human $I$ gene, the adult i phenotype, and the relationship with congenital cataracts. The most interesting deduction is that the I $\beta 6 \mathrm{GlcNAcT}$ activity of the human $I$ gene (possibly from the IGnTB gene) may play an essential role in maintaining lens transparency. It should be noted, however, that the lenses used for the RT-PCR analysis were from adults, and the question of whether the expression of IGnTB in the lens occurs during human embryonic development is left for future consideration. In addition, although the results of the present investigation are highly suggestive, direct evidence implicating an I-gene defect in the development of congenital cataracts is still lacking. The requisite evidence may be obtained through a gene knockout experiment in a mouse model. Further investigation to elucidate the functional role of I $\beta 6 \mathrm{GlcNAcT}$ activity for maintenance of lens transparency will be significant.

\section{References}

1. Neimann $\mathrm{H}$, Watanabe $\mathrm{K}$, Hakomori S, Childs RA, Feizi T. Blood group i and I activities of 'lacto $\mathrm{N}$-nor-hexaosylceramide' and its analogues: the structural requirements for i-specificity. Biochem Biophys Res Commun. 1978;81:1286-1293.

2. Watanabe K, Hakomori S, Childs RA, Feizi T Characterization of a blood group I-active ganglioside. J Biol Chem. 1979;254:3221-3228.

3. Koscielak J, Zdebska E, Wilczynska Z, MillerPodraza H, Dzierzkowa-Borodej W. Immunochemistry of li-activity glycosphingolipids. Eur J Biochem. 1979:96:331-337.

4. Daniels G. Human Blood Groups. Oxford, England: Blackwell Science; 1995.

5. Issitt PD, Anstee DJ. Applied Blood Group Serology. Durham, NC: Montgomery Scientific Publications; 1998.

6. Wiener AS, Unger LJ, Cohen L, Feldman J. Typespecific cold auto-antibodies as a cause of acquired hemolytic anemia and hemolytic transfusion reactions: biological test with bovine red cells. Ann Intern Med. 1956;44:221-240.

7. Jenkins WJ, Marsh WL, Noades J, Tippett $P$, Sanger R, Race RR. The I antigen and antibody. Vox Sang. 1960;5:97-106.

8. Tippett P, Noades J, Sanger R, et al. Furthe studies of the I antigen and antibody. Vox Sang. 1960;5:107-121.

9. Marsh WL, Jenkins WJ. Anti-i: a new cold antibody. Nature. 1960;188:753.

10. Marsh WL. Anti-i: a cold antibody defining the relationship in human red cells. $\mathrm{Br} \mathrm{J}$ Haematol. 1961;7:200-209.

11. Marsh WL, Nichols ME, Allen FH. Inhibition of anti-I sera by human milk. Vox Sang. 1970;18: 149-154.

12. Rouger P, Juszczak G, Doinel C, Salmon C. Relationship between I and $\mathrm{H}$ antigens. I. A study of the plasma and saliva of a normal population. Transfusion. 1980;20:536-539.

13. Reid ME, Lomas-Francis C. The Blood Group
Antigen FactsBook. San Diego, CA: Academic Press; 1997.

14. Fukuda M, Fukuda M, Hakomori S. Developmental change and genetic defect in the carbohydrate structure of band 3 glycoprotein of human erythrocyte membrane. J Biol Chem. 1979;254:37003703.

15. Piller F, Cartron J-P. Biosynthesis of blood group I antigens. J Biol Chem. 1984;259:13385-13390.

16. Marsh WL, Nichols ME, Reid ME. The definition of two I antigen components. Vox Sang. 1971;20: 209-217.

17. Hakomori S, Kannagi R. Glycosphingolipids as tumor-associated and differentiation markers. J Natl Cancer Inst. 1983;71:231-251.

18. Feizi T. Demonstration by monoclonal antibodies that carbohydrate structures of glycoproteins and glycolipids are onco-developmental antigens. Nature. 1985;314:53-56.

19. Yamaguchi H, Okubo Y, Tanaka M. A note on possible close linkage between the li blood locus and a congenital cataract locus. Proc Jpn Acad. 1972; 48:625-628.

20. Ogata H, Okubo Y, Akabane T. Phenotype i associated with congenital cataract in Japanese. Transfusion. 1979;19:166-168.

21. Okubo Y, Yamaguchi H. I-negative phenotype and cataract. In: Program and abstracts of the XIX Congress of the International Society of Blood Transfusion; 1986; Sidney, Australia; 1986: 147.

22. Lin-Chu M, Broadberry RE, Okubo Y, Tanaka M. The i phenotype and congenital cataracts among Chinese in Taiwan [letter]. Transfusion. 1991;31: 676-677.

23. Yu L-C, Twu Y-C, Chang C-Y, Lin M. Molecular basis of the adult I phenotype and the gene responsible for the expression of the human blood group I antigen. Blood. 2001;98:3840-3845.

24. Marsh WL, DePalma H. Association between the li blood group and congenital cataract. Transfusion. 1982;22:337-338.
25. Macdonald EB, Douglas R, Harden PA. A Caucasian family with the i phenotype and congenital cataracts. Vox Sang. 1983;44:322-325.

26. Page PL, Langevin S, Petersen RA, Kruskall MS Reduced association between the li blood group and congenital cataracts in white patients. Am J Clin Pathol. 1987;87:101-102.

27. Bierhuizen MFA, Mattei M-G, Fukuda M. Expres sion of the developmental I antigen by a cloned human cDNA encoding a member of a $\beta-1,6-N$ acetylglucosaminyltransferase gene family. Genes Dev. 1993;7:468-478.

28. Brun A, Gaudernack G, Sandberg S. A new method for isolation of reticulocytes: positive selection of human reticulocytes by immunomagnetic separation. Blood. 1990;76:2397-2403.

29. Bierhuizen MFA, Maemura K, Kudo S, Fukuda M. Genomic organization of core 2 and I branching $\beta$-1,6- $N$-acetylglucosaminyltransferase gene family. Glycobiology. 1995;5:417-425.

30. Yeh J-C, Ong E, Fukuda M. Molecular cloning and expression of a novel $\beta-1,6-N$-acetylglucosaminyltransferase that forms core 2 , core 4 and I branches. J Biol Chem. 1999;274:32153221.

31. Kadakol A, Ghosh SS, Sappal BS, Sharma G, Chowdhury JR, Chowdhury NR. Genetic lesions of bilirubin uridine-diphosphoglucuronate glucuronosyltransferase (UGT1A1) causing CriglerNajjar and Gilbert syndromes: correlation of genotype to phenotype. Hum Mutat. 2000;16:297306.

32. Dzierzkowa-Borodej W, Seyfried H, Nichols M Reid M, Marsh WL. The recognition of watersoluble I blood group substance. Vox Sang. 1970; 18:222-234

33. Marsh WL, Jensen L, Decary F, Colledge K. Water-soluble I blood group substance in the secretions of i adults. Transfusion. 1972;12:222 226.

\section{Erratum}

In the letter by Alvarez et al entitled "Cytogenetic characterization reveals that the SAM-1 erythroid cell line is derived from K-562 cells," which appeared in the November 1, 2002, issue of Blood (Volume 100:3435), the cell line previously reported to be a derivative of K562 should have been TI-1. 